\title{
Impact of a Novel Insulin Management Service on Non-insulin Pharmaceutical Expenses
}

\author{
John E. Schneider ${ }^{1}$, Anjani Parikh ${ }^{1 *}$, Ivana Stojanovic ${ }^{1}$ \\ ${ }^{1}$ Avalon Health Economics, Morristown, NJ, USA \\ *Corresponding author: anjani.parikh@avalonecon.com
}

\begin{abstract}
Background: Studies have shown that improvements in glycemic control are associated with avoidance or delayed onset of diabetes complications, improvements in health-related quality of life, and reductions in diabetes-related health care costs. Clinical practice guidelines recommend maintaining a hemoglobin A1c (HbA1c) level less than 7\%, but among type 2 diabetes patients using insulin, two-thirds have HbA1c above $7 \%$ and one-third have HbA1c above 9\%.
\end{abstract}

Objectives: This study examined the use of insulin management services to enable patients to optimize insulin dosing to achieve HbA1c targets and subsequently reduce health care costs. Cost savings may be achieved through reduced complications and hospitalizations, as well as reduced outpatient, physician, and clinic costs. This study quantified the reduction in pharmaceutical expenses related to the use of an enhanced insulin management service to improve glycemic control.

Methods: Two hundred seventeen insulin-reliant patients were enrolled in the d-Nav ${ }^{\circledR}$ Insulin Guidance Service through a participating insurance group. A prospective cost analysis was conducted using data from enrolled patients who completed the first 90 days of follow up.

Results: Of the 192 patients who completed the 90-day study period, 54 (28.13\%) were prescribed one or more expensive medications at baseline, but 45 (83.33\%) of those patients were eligible for medication discontinuation after 90 days. At baseline, the annual cost of expensive medications per patient was $\$ 7564$ (CI: \$5191-\$9938) and \$1483 (CI: -\$1463-\$4429) at 90 days $(\mathrm{p}<0.001)$. Direct savings from medication elimination was estimated to be $\$ 145$ per patient per month (PPPM) or $\$ 1736$ per patient per year (PPPY) for all patients and \$514 PPPM/\$6172 PPPY for the target group. Patients that completed the 90 -day period significantly reduced HbA1c levels from 9.37\% (CI:7.72\%-11.03\%) at baseline to 7.71\% (CI: 6.70\%-8.73\%) (p<0.001). A total of $170(88.54 \%)$ patients had improved HbA1c at 90 days.

Conclusions: Use of the insulin guidance service achieved improved glycemic control by optimizing insulin dosing, which enabled most patients using the service to reduce or eliminate the use of expensive diabetes medications. Further study is needed to assess the impact of optimized insulin dosing on other diabetes-related health care costs in a usual practice setting.

Keywords: Economic Evaluation; Diabetes; Insulin Management Device 


\section{Introduction}

Diabetes affects an estimated 30.3 million people in the U.S., representing nearly $10 \%$ of the general population; about $90-95 \%$ have type 2 diabetes (T2D). ${ }^{1}$ Numerous studies have shown that improvements in glycemic control are associated with avoidance or delayed onset of diabetes complications, improvements in patient health-related quality of life (HRQL), and reductions in diabetes-related health care costs.

Clinical practice guidelines for treating T2D recommend maintaining a hemoglobin A1c (HbA1c) level less than $7 \%$, though benefits of lower HbA1c have also been noted. ${ }^{2}$ However, HbA1c targets often are difficult to maintain in usual practice. In the U.S., among type 2 diabetes patients using insulin, two-thirds have HbA1c above $7 \%$, and one-third have HbA1c above $9 \% .{ }^{2}$ Poor glycemic control in the T2D population is associated with a number of complications, including ischemic heart disease, congestive heart failure, stroke, foot ulcers, amputation, blindness, renal failure, and poor wound healing. ${ }^{3}$ The likelihood of T2D patients experiencing these complications is closely related to their degree of diabetes control, with the likelihood of complications increasing significantly for each $1 \%$ increase in HbA1c. ${ }^{4}$ For example, one study examining the relationship of glycemic control and hypoglycemic episodes over a 4-year period found that each 1\% unit increase in average HbA1c from $9.3 \%$ was associated with a 31\% increase in the risk of cardiovascular death and a 36\% increase in the risk of stroke. ${ }^{5}$ In addition to reducing complications, improved glycemic control has also been shown to improve HRQL of diabetes patients, including improvement in symptom distress, cognitive functioning, general perceived health, and work productivity. ${ }^{6,7}$

The goal of insulin management services is to help patients optimize insulin dosing to enable them to achieve HbA1c targets and improve their HRQL. Improved glycemic control also reduces diabetes-related healthcare costs by reducing costs associated diabetes complications, such as hospitalizations and outpatient services. Optimized insulin dosing achieved through an insulin management service also may reduce overall drug costs by reducing the use of expensive diabetes medications. However, to date, the effect of insulin management services on the cost of care has only been examined within a decision model framework. ${ }^{8}$ The purpose of this study was to assess the ability of an insulin management service to reduce costs associated with the use of expensive diabetes drugs in a usual care setting within the U.S. health system.

\section{Background}

\section{Glycemic Management}

Good glycemic control is a well-accepted outcome for diabetes management, and clinical practice guidelines recommend a step-wise approach. ${ }^{9}$ Treatment typically begins with metformin, and if the HbA1c target is not achieved after 3 months, a second agent is added, such as sulfonylurea, thiazolidinedione, DPP-4 inhibitor, SGLT2 inhibitor, GLP-1 receptor agonist, or basal insulin. Due to increased risk of cardiovascular events, thiazolidinedione use has diminished in the past decade, and use of the other agents has increased. ${ }^{10,11}$ Specifically, costs associated with increased use of DPP-4 inhibitors, SGLT2 inhibitors, and GLP-1 receptor agonists has risen from $\$ 10$ billion to $\$ 22$ billion from 2002 to $2012 .{ }^{12}$ In addition, due to its progressive nature, many patients with T2D eventually require and benefit from insulin therapy, usually in combination with metformin. According to the standard of care, when a patient fails to reach their HbA1c target with dual therapy of metformin plus insulin, a third agent should be prescribed, such as thiazolidinedione, DPP-4 inhibitor, SGLT2 inhibitor, or GLP-1 receptor agonist. ${ }^{9}$ However, despite the wide availability of multiple classes of therapeutic agents, most insulin users have HbA1c well above the accepted targets and their

diabetes is uncontrolled. ${ }^{13}$ 


\section{Diabetes and Insulin}

Insulin is typically prescribed when a patient with advanced T2D becomes insulin deficient; it is one of the most commonly prescribed medications worldwide. In addition to insulin, many advanced T2D patients are prescribed a combination of oral anti-diabetics such as alpha-glucosidase inhibitors, biguanides, DPP-4 inhibitors, GLP-1 receptor agonists, meglitinides, SGLT-2, and sulfonylureas. ${ }^{14}$ Insulin formulations offer many benefits, including diverse pharmacodynamic profiles, no upper dosage limit, and only one source of toxicity (hypoglycemia). Despite the long-term availability and the potential advantages of insulin therapy, in practice its effectiveness is suboptimal. The average level of $\mathrm{HbA} 1 \mathrm{c}$ for patients treated with insulin has not improved for decades, and frequent titration of insulin dosage is crucial to maintain optimal glycemic control and avoid hypoglycemia. ${ }^{15}$ Optimizing insulin dosing among insulin-reliant individuals with T2D has been shown to be a very effective means of lowering $\mathrm{HbA1c}$ and achieving clinical targets. ${ }^{14,16}$

\section{Optimizing Insulin Dosing}

Multiple studies have emphasized the importance of frequent insulin titration in achieving glycemic goals. ${ }^{14,16-18}$ Dose titration, with frequent adjustments, has been shown to maximize treatment utility because metabolisms vary over time, beyond daily fluctuations in energy balance. ${ }^{14,16,19-22}$ However, the current structure of the health care system does not encourage (or in many cases permit) such frequent review of glucose data and corresponding timely insulin dosage modification. ${ }^{19}$ In usual practice, insulin dose adjustments are done every 90 to 180 days during an outpatient clinic visit. ${ }^{16}$ According to Khunti et al., "For most patients requiring insulin therapy, dose titration is carried out by physicians; however, evidence suggests that this process may not provide optimal glycemic management for patients." ${ }^{16}$ To date, target HbA1c levels have only been consistently maintained in clinical trial settings with frequent insulin titration and monitoring. ${ }^{21}$ This improvement lasted only as long as periodic insulin adjustments were made. According to Harper et al., the magnitude of intraindividual variability in insulin requirements is considerable and explains both bouts of hyperglycemia and hypoglycemia in insulin users. ${ }^{21}$

\section{Insulin Management Service}

The d-Nav ${ }^{\circledR}$ Insulin Guidance Service was designed to optimize insulin therapy by providing safe and effective personalized insulin dosage recommendations to T2D patients without increasing the workload of referring physicians. ${ }^{19,23}$ This service is a combination of dedicated health care professionals and technology designed to simplify and enhance insulin therapy. Patients referred to the service receive training and support from a clinical team to help them use the accompanying d-Nav device, followed by ongoing supervision of the process, including clinical triaging to alert referring physicians in the case of abnormal disease progression. Patients use the device to monitor glucose levels before each injection and it then provides them with a personalized dose recommendation. By analyzing glucose patterns, the device automatically adjusts insulin dosage, as often as needed, to achieve and maintain optimal glycemic balance for each individual. This allows patients to adjust insulin therapy to fit their changing needs while preventing hypoglycemia. In several studies, the d-Nav Insulin Guidance Service has been shown to improve glycemic control, reduce hypoglycemia frequency, and reduce costs. ${ }^{19,24}$ As of May 2016, 727 patients have been referred to the service in Northern Ireland, and 533 patients have used it as of mid-May 2016. Among these active users, 47 patients have used the service for more than three years, and 103 have used it for more than two years. Average HbA1c improved from $9.5 \%( \pm 1.6 \%)$ to just over $7 \%$, and the lower rates were maintained throughout study periods. These results have been remarkably consistent in all studies of the service to date..$^{20,24-26}$ 


\section{Methods}

The objective of this study was to examine the cost differences within a cohort of T2D patients who use insulin, assessing the longitudinal changes from baseline [i.e., patient entering study treated under the "standard of care" (SOC)] to 90 days from starting the service. Patients were identified and referred by physicians from primary care and secondary care clinics in Southeastern Michigan. Adults (21-75 years of age) with T2D who were using insulin to manage their diabetes were eligible to participate if they had a $\mathrm{HbA} 1 \mathrm{c} \geq 7.5 \%$, and were insured by Blue Cross Blue Shield of Michigan. Patients were excluded from the trial if they had a body mass index of $\geq 45 \mathrm{~kg} / \mathrm{m}^{2}$; severe impairment of cardiac, hepatic, or renal functions; psychological or cognitive impairment; more than two episodes of severe hypoglycemia in the past year; or a history of hypoglycemia unawareness when glucose levels are $\leq 50 \mathrm{mg} / \mathrm{dl}$. The d-Nav device used for this study is CE-marked and has been in commercial use in the United Kingdom since October 2012. The costs of non-insulin diabetes drugs were collected over the initial 90-day period, as were changes in HbA1c levels as a secondary endpoint.

\section{Study Design}

For patients in the d-Nav arm, after they signed an informed consent form and were enrolled in the study, they received training on the proper use of the $\mathrm{d}-\mathrm{Nav}$ device, and a baseline $\mathrm{HbA} 1 \mathrm{c}$ level was obtained. At the end of the 90-day study period, patients returned to the care center for an HbA1c test. If the 90-day HbA1c was lower than the baseline HbA1c, then glycemic medications (other than insulin and metformin) were discontinued. All patients enrolled in the study were using one of four insulin regimens: (1) treated with the long-acting insulin analog Glargine $\left(\operatorname{Lantus}^{\circledR}\right.$ ); (2) treated with twice daily biphasic insulin (i.e., Humalog ${ }^{\circledR}$ Mix 75/25, NovoLog ${ }^{\circledR}$ Mix 70/30) or pre-mixed insulin (i.e., Humulin ${ }^{\circledR} 70 / 30$, Novolin ${ }^{\circledR} 70 / 30$ ); (3) treated with a short-acting insulin analog (i.e., Lispro-Humalog ${ }^{\circledR}$, Aspart-NovoLog ${ }^{\circledR}$, Glulisine-Apidra $^{\circledR}$ ) before each meal combined with the long-acting insulin analog Glargine; and (4) treated with a short-acting insulin analog before each meal utilizing an insulin/carbohydrate ratio for calculating their short-acting insulin doses, combined with the long-acting insulin analog Glargine.

After the initial visit was completed, patients were contacted via telephone at 2 days, 2 weeks, and 4 weeks to verify that patients were using d-Nav correctly and adhering to d-Nav recommended insulin dosing. Patients were withdrawn from the trial if (1) they wanted to stop utilizing the guidance service; (2) they were lost to follow-up; (3) switched insulin types to unsupported insulin; or (4) other health reasons. Patients who were withdrawn from the study were referred back to the SOC.

\section{Main Outcome Measures}

The main outcome measure for this study was costs of care for a specified set of non-insulin diabetes related prescription drugs, with particular focus on the costs of GLP-1 receptor agonists drugs, DPP-4i drugs, and SGLT-2i drugs (hereinafter labeled as "expensive" branded diabetes medications). Diabetes drugs (other than insulin) used by each patient at baseline were obtained at baseline and at the end of the 90-day intervention period. Drug costs were calculated using the prevailing market price for each drug. ${ }^{27}$ The analysis focused on the mean value for pre- and post-levels of costs for expensive diabetes drugs, and the mean difference in these drug costs over the study period. The costs of insulin were excluded from this analysis for two reasons: (1) costs of insulin vary greatly by brand; and (2) insulin costs are volume dependent. Both parameters are outside of the control of the intervention. In addition, because the price of insulin is volume-based and there is no steady state for insulin dosage, the cost of insulin during the 9-month intervention may not reflect long- or short-term projected costs of insulin. ${ }^{21}$ In addition to costs, two secondary outcomes of interest were also assessed: change in HbA1c and patient satisfaction. To evaluate if patients achieved reduction in 
HbA1c levels during the study period, we determined the difference between baseline HbA1c and the HbA1c measured at 90 days for each patient using a two-tailed student's t-test in Microsoft Excel. To assess patient satisfaction, surveys were mailed to everyone who completed the 90-day visit for the study. Participants were asked a series of questions regarding their perception and satisfaction with the service and were also asked how their satisfaction compared to the period of time prior to starting the service.

\section{Results}

The study recruited a total of 217 patients, of which 192 patients completed the 90-day visit and were available for analysis. Reasons for withdrawal for the 25 patients that did not complete the 90-day visit included (1) withdrawn consent ( $\mathrm{n}=12 ; 48 \%$ ); (2) lost to follow-up ( $\mathrm{n}=10 ; 40 \%)$; (3) switched insulin types to unsupported insulin ( $n=2 ; 8 \%)$; and (4) experienced a health complication unrelated to diabetes $(n=1 ; 4 \%)$.

\section{Use of Expensive Medications}

Of the 217 patients enrolled in the study, 57 (26.27\%) were prescribed expensive medications at enrollment. Of the 57 patients, $3(12.50 \%$ ) withdrew before the 90 -day visit. Therefore, of the 192 patients who completed the study, $54(28.13 \%)$ were prescribed at least one expensive medication at time of enrollment. Of these, 27 (50.0\%) were prescribed GLP-1 receptor agonists, 17 (31.5\%) were prescribed DPP-4i drugs, and 13 (24.07\%) were prescribed SGLT-2i drugs. Two were prescribed with more than one of these expensive medications, and one was prescribed with a combination of a DPP-4i and an SGLT-2i.

\section{Medication Discontinuation}

Of the 192 patients that completed the 90-day visit, 170 (88.54\%) had improved glycemic control and were therefore eligible for medication discontinuation. Of the 54 patients on one or more expensive medications, 45 $(83.33 \%)$ were eligible for discontinuation based on improvement of their HbA1c. Among those 45 patients, the majority were taking GLP-1 or DPP-4 drugs (Table 1).

Table 1. Patients Prescribed and Taking Expensive Medications but Eligible for Discontinuation

\begin{tabular}{lcccc}
\hline Drug Class & $\begin{array}{c}\text { \% of Patients (n=54) } \\
(\mathbf{e})\end{array}$ & $\begin{array}{c}\text { \% Eligible for } \\
\text { Discontinuation } \\
\text { (f) }\end{array}$ & $\begin{array}{c}\text { Number of } \\
\text { Prescriptions } \\
\text { During } \\
\text { Enrollment } \\
\text { (g) }\end{array}$ & $\begin{array}{c}\text { Prescriptions Eligible for } \\
\text { Discontinuation (h) }\end{array}$ \\
\hline GLP-1 (a) & $50.00 \%$ & $85.19 \%$ & 27 & 23 \\
\hline DPP-4 (b)(d) & $31.48 \%$ & $82.35 \%$ & 17 & 14 \\
\hline SGLT-2 (c)(d) & $24.07 \%$ & $69.23 \%$ & 13 & 9 \\
\hline Total & NA (i) & $83.33 \%$ & 57 & 46 \\
\hline
\end{tabular}

Note: (a) GLP-1a drugs include Bydureon, Victoza, Byetta, and Trulicity; (b) DPP-4i drugs include Janumet and Januvia; (c) SGLT-2i drugs include Invokana, Farxiga, Invokamet, and Jardiance; (d) One patient was on Glyxambi, a combination DPP-4 and SGLT2 drug and was counted for both drug classes for the purposes of this analysis; (e) Refers percentage of all patients who are on expensive drugs; (f) Out of all prescriptions categorized with this class of drugs, calculation of columns (h)/(g); (i) does not sum to $100 \%$ because of patients on more than one expensive drug. 


\section{Economic Effects}

Based on discontinuation rates applied to data on drug costs, the direct savings from the elimination of medications are expected to be approximately $\$ 145$ per participant per month (PPPM) or $\$ 1736$ per participant per year (PPPY) for all patients and \$514 PPPM/ \$6172 PPPY for the target group ( $\mathrm{n}=54$ ) (Table 2). There is a considerable range in costs depending on specific drug regimens. For example, for patients taking Victoza $(\mathrm{n}=12)$, monthly savings from discontinuation would be $\$ 832$ PPPM and \$9984 PPPY, and for patients prescribed Bydureon, the next most commonly prescribed brand-name drug $(n=10)$, monthly savings from discontinuation would be \$644 PPPM and \$7728 PPPY (data not shown in table). Over the course of the study period, reductions in average 12-month medication cost savings for patients in the target group ( $\mathrm{n}=54$ ) was $\$ 7564$ (CI: \$5190-\$9938) at setup and \$1483 (CI: $-\$ 1463$ - \$4429) at the end of the 90-day period ( $<<0.001)$. Reductions in average 12-month costs for all patients $(\mathrm{n}=192)$ at enrollment was $\$ 2362$ (CI: $-\$ 1254-\$ 5978)$ and $\$ 587$ (CI: $-\$ 1178-\$ 2352)(\mathrm{p}<0.01)$ at 90 days. In sum, it is clear that the cost savings associated with improved glycemic control are substantial and likely to result in substantial savings over a relatively short period of time.

Table 2. Comparison of Monthly and Annual Cost Savings for All Patients and Target Group Patients

\begin{tabular}{lcc}
\hline \multicolumn{1}{c}{ Patient Group } & Monthly Cost Savings (a) & Annual Cost Savings \\
\hline All Patients $(\mathrm{n}=192)$ & $\$ 145$ & $\$ 1736$ \\
\hline Target Group $(\mathrm{n}=54)$ (b) & $\$ 514$ & $\$ 6172$ \\
\hline
\end{tabular}

Notes: (a) Average monthly cost per patient; prices are reported by GoodRx.com and are based on market value as of October 2017; (b) Target Group represents cost savings for patients taking at least one expensive medication (either GLP-1a, DPP-4i, or SGLT-2i)

\section{Improved Glycemic Control}

Table 3. Effect of d-Nav on Patient HbA1c Levels at 90-days ( $\mathrm{n}=192)$

\begin{tabular}{lccc}
\hline \multicolumn{1}{c}{ Patient Cohort } & $\begin{array}{c}\text { Average HbA1c at } \\
\text { Enrollment }\end{array}$ & $\begin{array}{c}\text { Average HbA1c } \\
\text { after } \mathbf{3} \text { months of } \\
\mathbf{d - N a v}\end{array}$ & $\begin{array}{c}\text { Percentage Point } \\
\text { Difference (a) }\end{array}$ \\
\hline All patients $(\mathrm{n}=192 ; 100.00 \%)$ & $9.37 \%$ & $7.71 \%$ & $-1.66(\mathrm{p}<0.001)^{* *}$ \\
\hline Target Group $(\mathrm{n}=54 ; 28.13 \%)$ & $9.30 \%$ & $7.86 \%$ & $-1.44(\mathrm{p}<0.001)^{* *}$ \\
\hline HbA1c $>\mathbf{9 \%}$ at baseline $(\mathrm{n}=\mathbf{9 8 ;} \mathbf{5 1 . 0 4 \% )}$ & & & \\
\hline HbA1c $\leq 9 \%(\mathrm{n}=84 ; 85.71 \%)$ & $10.49 \%$ & $7.63 \%$ & $-2.86(\mathrm{p}<0.001)^{* *}$ \\
\hline HbA1c $>9 \%(\mathrm{n}=14 ; \mathbf{1 4 . 2 9 \% )}$ & $11.05 \%$ & $10.20 \%$ & $-0.85(\mathrm{p}=0.04)^{*}$ \\
\hline
\end{tabular}

Notes: Percentage point difference between baseline and 3-month assessment. See text.

$*$ Significant at the $95 \%$ level

$* *$ Significant at the $99 \%$ level

Average HbA1c for the 192 patients who completed the 90-day period improved from 9.37\% (CI: 7.72-11.03) at baseline to $7.71 \%$ (CI: $6.70-8.73)$ by 1.66 percentage points $(\mathrm{p}<0.001)$. Average HbA1c for the target group patients on expensive medications $(n=54)$ improved by 1.43 percentage points from $9.30 \%(\mathrm{CI}: 7.7 \%-10.9 \%)$ at baseline to $7.86 \%(\mathrm{CI}: 6.81 \%-8.91 \%)(\mathrm{p}<0.001)$ at 90 -days. A separate analysis was applied for the subgroup of patients who had HbA1c $>9 \%$ at baseline, usually referred to as "poorly controlled" diabetes. Of the 192 patients who completed the 90-day visit, $98(51.04 \%)$ had a baseline HbA1c > 9\%. Of these, $84(85.71 \%)$ had a 90-day HbA1c $\leq 9 \%$. This subgroup of 97 patients saw an average decrease of 2.86 percentage points in HbA1c, from $10.49 \%$ at baseline to $7.63 \%$ at the 90 -day visit $(\mathrm{p}<0.001)$ (Table 3$)$. Those patients who still had $\mathrm{HbA} 1 \mathrm{c}>9 \%$ at the 90 -day visit still experienced a smaller but significant decline of 0.85 percentage points. 


\section{Patient Satisfaction}

As a secondary outcome endpoint, patient satisfaction among users of the insulin management service was also assessed. Surveys were mailed to everyone who completed the 90-day visit for the study; 75 surveys were received in the first round of mailings, and 28 were received in the second round of mailings, for a total of 103 respondents. The average item-level response rate was greater than $98 \%$. The questionnaire consisted of three sections: two asked participants about their overall satisfaction with their control of diabetes and the service, and a third asked about patient perception of diabetes management prior to starting the service. Participants were asked to score each question as follows: 1 = Strongly Agree; 2 = Somewhat Agree; $3=$ Somewhat Disagree; 4 = Strongly Disagree; and DK = Don't Know.

The mean score (and standard deviation) for key questions were as follows: "More satisfied with ability to manage diabetes" 1.31 (0.56); "Confident that d-Nav is using blood sugar results to change insulin dose" 1.26 (0.52); "Satisfied with decision to start using the d-Nav service" 1.17 (0.47); and "Important for patient to be able to continue using the d-Nav service" 1.22 (0.50). Overall, for all questions regarding the d-Nav study period, the vast majority of participants rated the service favorably, with the majority of responses either "Strongly Agree" or "Somewhat Agree." For the questions regarding the period before using the service, participants generally ranked their disease management as less effective [e.g., "Satisfied with ability to manage diabetes" $3.08(0.86)]$.

\section{Discussion}

This study found that average annual cost of expensive medications by patients was \$7564 (CI: \$5191-\$9938) and \$1483 (CI: \$-1463-\$4429) ( $\mathrm{p}<0.001)$. The direct savings from the elimination of medications alone estimated to be \$145 PPPM or \$1736 PPPY for all patients and \$514 PPPM/\$6172 PPPY in the target group of patients who were on expensive medications. Thus, for a health plan with 10,000 T2D "target group" patients, the estimated annual savings from medication discontinuation alone would be nearly $\$ 62$ million. In addition to these cost savings, among the 192 patients who completed the 90 -day period, there was a significant reduction in $\mathrm{HbA} 1 \mathrm{c}$ from $9.37 \% \pm 1.66 \%$ at baseline to $7.71 \% \pm 1.02 \%(\mathrm{p}<0.001)$ at the 90 -day visit. This level of clinical improvement is likely to generate substantial additional savings beyond medication discontinuation. ${ }^{4,7,28,29}$ And it is clear from the results of the satisfaction survey that participants perceived that their disease management had improved markedly while using the service, with no reported disutility associated with use of the device and service.

These savings are likely an understatement of the savings that could be achieved by combining the insulin guidance service with a cost-efficient insulin utilization protocol. Note that 1000 units of Novolin $70 / 30$ costs about $\$ 24$, while 1000 units of Tresiba costs $\$ 308$ and 1000 units of Humalog costs $\$ 353$. Accordingly, if a patient takes 100 insulin units per day of basal-bolus insulin analogs, the monthly costs would be almost $\$ 1000$. If that patient switches to Novolin 70/30 and doubles their insulin dose to 200 units per day, the monthly costs of insulin would still be only $\$ 144 .{ }^{27}$ Data provided in private communication by providers in the UK cohort of service users suggested that outcomes for patients on the service utilizing twice daily insulin therapy are slightly better than outcomes for service users who utilize basal-bolus insulin therapy, suggesting that implementing such an insulin utilization protocol would not compromise patient care.

Indirect effects are also likely to add to the direct service-related savings found in our study. Juarez et al., for example, suggested that approximately $30 \%$ of insulin users exhibit poorly controlled diabetes, ${ }^{30}$ and pay-for-performance measures such as Medicare STAR or HEDIS rewards providers if the vast majority of their patients have HbA1c $\leq 9 \%$. Moreover, glycemic control is one of the standard measures that 
comprise the Center for Medicare \& Medicaid Services' (CMS) 5-star scoring system for performance-based bonuses to Medicare Advantage (MA) plans. ${ }^{31} \mathrm{Li}$ and Doshi, for example, found that if a health plan is able to increase its composite CMS score by 1 star, that 1-star increase would be associated with an added 11337 enrollees $(\mathrm{p}<0.001) .{ }^{32}$ This translates to a marginal enrollment increase of $12.3 \%$ (not reported in published paper; obtained via personal communication with authors). Thus, for a health plan with 1000000 enrollees, a 1 -star increase is associated with a gain of 123000 enrollees. Assuming an average annual insurance premium of $\$ 12000$, in this example the 1 -star increase would lead to a $\$ 1.48$ billion increase in revenue. This excludes the actual value of the bonus payment to the plan, which would be substantial: in 2016, for example, Cigna earned a bonus of more than $\$ 250$ million. ${ }^{33}$ Another indirect effect is return-to-work and workplace productivity measures, such as presenteeism. While these outcomes were not directly assessed, they are likely to be positively impacted by the improved glycemic control. ${ }^{34-36}$

There are some expected limitations to the findings of this study. First, because this study involves the use of a medical device, patient adherence or lack thereof may impact HbA1c levels and overall study outcomes. Moreover, direct cost savings may also vary depending on cost of medication in different markets. Further follow-up research is recommended to better understand the long-term impact of the insulin guidance service on healthcare cost.

\section{Conclusions}

An enhanced insulin management service provided substantial cost savings from improved glycemic control related to insulin dosing optimization and the associated reduction in utilization of expensive diabetes medications. We found that the direct savings from the elimination of expensive medications alone are expected to be approximately \$145 PPPM or \$1736 PPPY for all patients and \$514 PPPM/\$6172 PPPY for the target group. Marked improvements in glycemic control, as evidenced by the significant drop in HbA1c over the study period, are also likely to generate additional savings in diabetes-related medical care costs beyond savings from discontinuation of expensive drugs. These direct medical care savings are likely to understate total savings, such as savings associated with improved workplace productivity. In addition to cost saving, the use of an insulin utilization protocol to improved glycemic control could improve facility and health-plan level quality metrics, contributing to facility and health-plan revenues through higher payments based on quality performance.

\section{References}

${ }^{1}$ Centers for Disease Control and Prevention. National Diabetes Statistics Report, 2017. In. Atlanta, GA: U.S. Dept of Health and Human Services; 2017.

${ }^{2}$ Qaseem A, Vijan S, Snow V, Cross JT, Weiss KB, Owens DK. Glycemic control and type 2 diabetes mellitus: the optimal hemoglobin A1c targets. A guidance statement from the American College of Physicians. Ann Intern Med. 2007;147(6):417-422.

${ }^{3}$ Blondet JJ, Beilman GJ. Glycemic control and prevention of perioperative infection. Curr Opin Crit Care. 2007;13(4):421-427.

${ }^{4}$ Menzin J, Korn JR, Cohen J, et al. Relationship between glycemic control and diabetes-related hospital costs in patients with type 1 or type 2 diabetes mellitus. J Manag Care Pharm. 2010;16(4):264-275.

${ }^{5}$ Freemantle N, Danchin N, Calvi-Gries F, Vincent M, Home PD. Relationship of glycaemic control and hypoglycaemic episodes to 4-year cardiovascular outcomes in people with type 2 diabetes starting insulin. Diabetes Obes Metab. 2016;18(2):152-158. 
${ }^{6}$ Testa MA, Simonson DC. Health economic benefits and quality of life during improved glycemic control in patients with type 2 diabetes mellitus: a randomized, controlled, double-blind trial. JAMA. 1998;280(17):14901496.

${ }^{7}$ Fidler C, Elmelund Christensen T, Gillard S. Hypoglycemia: an overview of fear of hypoglycemia, quality-oflife, and impact on costs. J Med Econ. 2011;14(5):646-655.

${ }^{8}$ Green W, Taylor M. Cost-effectiveness analysis of d-Nav for people with diabetes at high risk of neuropathic foot ulcers. Diabetes Ther. 2016;7(3):511-525.

9 American Diabetes Association. Pharmacologic approaches to glycemic treatment. Sec. 8. In Standards of Medical Care in Diabetes-2017. 2017. 0149-5992.

${ }^{10}$ Shaya FT, Lu Z, Sohn K, Weir MR. Thiazolidinediones and cardiovascular events in high-risk patients with type-2 diabetes mellitus: a comparison with other oral antidiabetic agents. J Clin Pharm Ther. 2009;34(9):490501.

${ }^{11}$ Jain R, Mullins CD, Lee H, Wong W. Use of rosiglitazone and pioglitazone immediately after the cardiovascular risk warnings. J Soc Adm Pharm. 2012;8(1):47-59.

${ }^{12}$ McEwen LN, Casagrande SS, Kuo S, Herman WH. Why are diabetes medications so expensive and what can be done to control their cost? Curr Diab Rep. 2017;17(9):71.

${ }^{13}$ Carrington MJ, Cohen N, Wiley JF. Blood glucose levels and glycaemic burden in 76,341 patients attending primary care: Bittersweet findings from a 9-year cohort study. Diabetes Res Clin Pract. 2017;127(Supplement C):89-96.

${ }^{14}$ Lovre D, Fonseca V. Benefits of timely basal insulin control in patients with type 2 diabetes. $J$ Diabetes Complications. 2015;29(2):295-301.

${ }^{15}$ Davidson MB. How our current medical care system fails people with diabetes. Diabetes Care. 2009.

${ }^{16}$ Khunti K, Davies MJ, Kalra S. Self-titration of insulin in the management of people with type 2 diabetes: a practical solution to improve management in primary care. Diabetes Obes Metab. 2013;15(8):690-700.

${ }^{17}$ Crasto W, Jarvis J, Khunti K, Davies MJ. New insulins and new insulin regimens: a review of their role in improving glycaemic control in patients with diabetes. Postgrad Med J. 2009;85(1003):257-267.

${ }^{18}$ Shaefer CF, Anderson J. The importance of postprandial glycemic control: optimizing add-on therapy to basal insulin. Postgrad Med. 2016;128(1):137-144.

${ }^{19}$ Bashan E, Harper R, Bi Y, Hodish I. A novel approach to optimise glycaemic control in insulin users. BMJ Case Rep. 2015;2015.

${ }^{20}$ Bashan E, Hodish I. Frequent insulin dosage adjustments based on glucose readings alone are sufficient for a safe and effective therapy. J Diabetes Complications. 2012;26(3):230-236.

${ }^{21}$ Harper R, Donnelly R, Bi Y, Bashan E, Minhas R, Hodish I. Dynamics in insulin requirements and treatment safety. J Diabetes Complications. 2016.

${ }^{22}$ Selvin E, Parrinello CM, Daya N, Bergenstal RM. Trends in insulin use and diabetes control in the U.S.: 1988-1994 and 1999-2012. Diabetes Care. 2016;39(3):e33-e35.

${ }^{23}$ Donelly R CS, Harper R. Diabetes insulin guidance system: A real-world evaluation of new technology (d-Nav) to achieve glycaemic control in insulin-treated type 2 diabetes. Practical Diabetes 2015.

${ }^{24}$ Harper R. Insulin Therapy Transformation in Northern Ireland. American Diabetes Association 2016.

${ }^{25}$ Bashan E, Herman WH, Hodish I. Are glucose readings sufficient to adjust insulin dosage? Diabetes Technol Ther. 2011;13(1):85-92. 
${ }^{26}$ Herman WH, Ilag LL, Johnson SL, et al. A clinical trial of continuous subcutaneous insulin infusion versus multiple daily injections in older adults with type 2 diabetes. Diabetes Care. 2005;28(7):1568-1573.

${ }^{27}$ GoodRx I. GoodRx Market Prices. 2017; https://www.goodrx.com. Accessed September 7, 2017.

${ }^{28}$ Banerji MA, Dunn JD. Impact of glycemic control on healthcare resource utilization and costs of type 2 diabetes: current and future pharmacologic approaches to improving outcomes. Am Health Drug Benefits. 2013;6(7):382-392.

${ }^{29}$ Gilmer TP, O’Connor PJ, Rush WA, et al. Predictors of health care costs in adults with diabetes. Diabetes Care. 2005;28(1):59-64.

${ }^{30}$ Juarez DT, Ma C, Kumasaka A, Shimada R, Davis J. Failure to reach target glycated a1c levels among patients with diabetes who are adherent to their antidiabetic medication. Popul Helath Manag. 2014;17(4):218223.

${ }^{31}$ Linehan K. Medicare Advantage update: benefits, enrollment, and payments after the ACA. Issue brief (George Washington University National Health Policy Forum). 2013(850):1-12.

${ }^{32} \mathrm{Li}$ P, Doshi JA. Impact of medicare advantage prescription drug plan star ratings on enrollment before and after implementation of quality-related bonus payments in 2012. PloS One. 2016;11(5):e0154357.

${ }^{33}$ Galewitz P. Medicare Plans Score Higher Ratings And Millions In Bonuses. Kaiser Health News;2016.

${ }^{34}$ Brod M, Wolden M, Christensen T, Bushnell DM. Understanding the economic burden of nonsevere nocturnal hypoglycemic events: impact on work productivity, disease management, and resource utilization. Value Health. 2013;16(8):1140-1149.

${ }^{35}$ Hex N, Bartlett C, Wright D, Taylor M, Varley D. Estimating the current and future costs of Type 1 and Type 2 diabetes in the UK, including direct health costs and indirect societal and productivity costs. Diabet Med. 2012;29(7):855-862.

${ }^{36}$ Lopez JM, Annunziata K, Bailey RA, Rupnow MF, Morisky DE. Impact of hypoglycemia on patients with type 2 diabetes mellitus and their quality of life, work productivity, and medication adherence. Patient Prefer Adherence. 2014;8:683-692. 